OHCA resuscitation. We investigated the recruiting process and characteristics of the lay volunteers.

Method From September 1 st 2017, lay-persons have been able to download the 'HeartRunner'-application and register as a lay volunteer in the Capital Region of Denmark comprising 1.8 million inhabitants. Recruiting strategies included advertisements outdoor and in newspapers, e-information, and through nationwide television coverage.

Results During the first four months, 14935 people registered as lay volunteers $(=820 / 100,000$ inhabitants $) ; 52.3 \%$ were male and $28.3 \%$ were health care professionals. Median age at registration was 36 years (27-48). In total, 278 suspected OHCAs were registered and 3029 lay volunteers were alerted (mean of 11 volunteers per alarm). Of all alerted lay volunteers, $51.3 \%(n=1,554)$ responded to the alarm and $56.9 \%$ $(n=884)$ accepted the mission. Of them, $53.7 \%$ were male, $32.0 \%$ were health care professionals, and median age was 38 years (27-49). The most effective recruiting strategy was nationwide television broadcast, which recruited nearly 6000 volunteers in one day.

Conclusion Lay-persons can be recruited through a smartphone application based response system, and a high proportion accepts to volunteer as first responder in OHCA resuscitation. Conflict of interest None

Funding Dr. L. Andelius is supported by a fund from The Danish foundation TrygFonden, who has no influence on study design; in the collection, analysis, or interpretation of data.

\section{SPATIOTEMPORAL AED OPTIMISATION IS GENERALIZABLE}

${ }^{1} \mathrm{CLF}$ Sun*, 2,3 K Karlsson, ${ }^{4} \mathrm{C}$ Torp-Pedersen, ${ }^{2,3} \mathrm{~F}$ Folke, ${ }^{1,5} \mathrm{TCY}$ Chan. ${ }^{1}$ Department of Mechanical and Industrial Engineering, University of Toronto, Canada; ${ }^{2}$ Emergency Medical Services Copenhagen, University of Copenhagen, Denmark; ${ }^{3}$ Department of Cardiology, Copenhagen University Hospital Gentofte, Hellerup, Denmark; ${ }^{4}$ The Department of Health Science and Technology, Aalborg University, Aalborg, Denmark; ${ }^{5}$ Rescu, Li Ka Shing Knowledge Institute, St. Michael's Hospital, Canada

\subsection{6/10.1136/bmjopen-2018-EMS.82}

Aim Mathematical optimisation of automated external defibrillator (AED) placements has the potential to improve out-ofhospital cardiac arrest (OHCA) coverage and reverse the negative effects of limited AED accessibility. However, the generalizability of optimisation approaches has not yet been investigated.

Method We examined the performance and generalizability of a spatiotemporal AED placement optimisation methodology, initially developed for Toronto, Canada, ${ }^{1}$ to the new study setting of Copenhagen, Denmark. We identified all atraumatic treated public OHCAs (1994-2016) and all registered AEDs (2016) in Copenhagen, Denmark. We then calculated the coverage loss associated with limited temporal accessibility of registered AEDs, and used a spatiotemporal optimisation model to quantify the potential coverage gain of optimised AED deployment. Coverage gain of spatiotemporal deployment over a spatial-only solution was quantified through 10 -fold crossvalidation. Statistical testing was performed using $\chi 2$ and McNemar's tests.

Results We identified 2149 public OHCAs and 1573 registered AED locations. Coverage loss was found to be $24.4 \%(1,104$ OHCAs covered under assumed $24 / 7$ coverage, and 835 OHCAs under actual coverage). The relative coverage gain from using the spatiotemporal model over a spatial-only approach was $15.3 \%$. Temporal and geographical trends in coverage gain were similar to Toronto.

Conclusion Without modification, a previously developed spatiotemporal AED optimisation approach was applied to Copenhagen, resulting in similar OHCA coverage findings as Toronto, despite large geographic and cultural differences between the two cities. In addition to reinforcing the importance of temporal accessibility of AEDs, these similarities demonstrate the generalizability of optimisation approaches to improve AED placement and accessibility.

\section{REFERENCE}

1. Sun CLF, Demirtas D, Brooks SC, Morrison LJ, Chan TCY. Optimising public defibrillator deployment to overcome spatial and temporal accessibility barriers. Journal of the American College of Cardiology 2016.

\section{Conflict of interest None}

Funding This work was funded by the ZOLL Foundation (ZOLL Foundation Research Grant) and supported by the Danish foundation TrygFonden with no commercial interest in the field of cardiac arrest.

\section{FOR OUT-OF-HOSPITAL CARDIAC ARREST (OHCA) AT PUBLIC LOCATIONS COMMUNITY BYSTANDER DEFIBRILLATION RESCUE HAS BETTER IMPACT ON PATIENT OUTCOMES COMPARED WITH DISPATCHER- ASSISTED TELEPHONE CPR (DATCPR)}

${ }^{1} \mathrm{PC} \mathrm{Ko*},{ }^{2} \mathrm{SC}$ Huang, ${ }^{3} \mathrm{YW}$ Chen, ${ }^{3} \mathrm{HY}$ Hsaio, ${ }^{4} \mathrm{CL}$ Shih. ${ }^{1}$ National Taiwan University Hospital, Taiwan; ${ }^{2}$ Taipei City Health Department, Taiwan; ${ }^{3}$ Taipei City Fire Department, Taiwan; ${ }^{4}$ Ministry of Health and Welfare, Taiwan

\subsection{6/10.1136/bmjopen-2018-EMS.83}

Aim We compared the outcomes between a community-wide bystander defibrillation rescue program and a DATCPR program in patients after out-of-hospital cardiac arrest at public sites.

Method A prospective 2-year community-wide observational database collected from a metropolitan OHCA Web-based Registry was studied, after a citywide bystander defibrillation rescue program had been launched that public accessed AEDs (automated external defibrillators) were strategically implemented in designated locations and electronically registered; and a DATCPR program had been well run in the dispatch centre. The survival outcomes of OHCA at pubic locations between the two program interventions were compared. Outcomes included 2-hour sustained ROSC (return of spontaneous circulation) at hospital, survival to hospital discharge, and good CPC (Cerebral Performance Category Scale 1 or 2). All patient prehospital characteristics and outcome relations were evaluated and adjusted by regression analysis.

Results The density of public AEDs distribution increased from 3.96 to 6.24 per square kilometres in the studied 2 years. Among a total of 6,356 OHCA, 627 patients occurred at public locations, including 28 patients (male for $82 \%$, witnessed arrest for 79\%) received bystander aid by public AEDs plus CPR rescue and 243 patients (male for 64\%, witnessed arrest for $61 \%$ ) received DATCPR intervention. For these 28 patients, $53.6 \%(15 / 28)$ achieved prehospital ROSC at scene or during transport, $71.4 \%(20 / 28)$ achieved sustained ROSC after resuscitation at hospital, $57.1 \%(16 / 28)$ achieved survival-to-discharge and noticeably all those $16(100 \%, 16 / 16)$ survival-to-discharge patients achieved excellent neurological outcome of CPC 1 (CPC Scale 1). Their outcomes were 